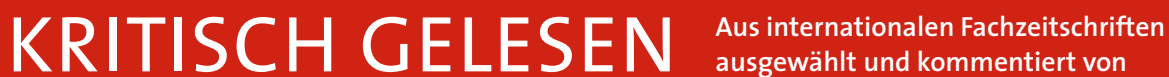

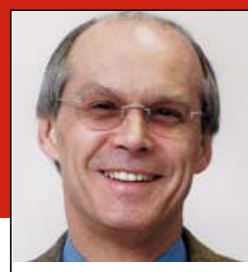

Prof. Dr. med. H. S. FüeßI Isar-AmperKlinikum, KI. MünchenOst, Haar

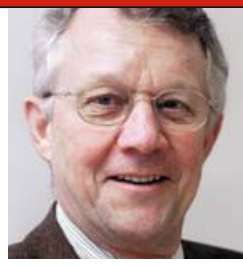

Prof. Dr. med. H. Holzgreve Internist, Kardiologische Praxis, München

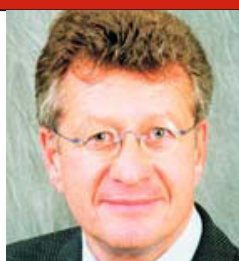

Dr. med. P. Stiefelhagen

Westerwald

Krankenhaus, Hachenburg

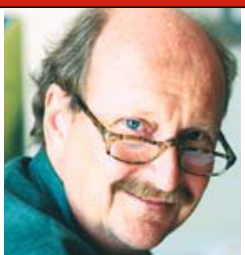

Prof. Dr. med.

E. Ernst

Peninsular

Medical School,

University of Exeter/UK

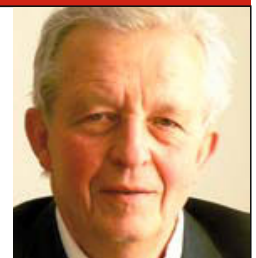

Prof. Dr. med. K. Malberg Immunologie, DresdenLoschwitz

\section{Gibt es eine Diät, die vor Alzheimer schützt?}

\begin{abstract}
Salate, Gemüse, Nüsse, frisches Obst und Geflügel sollen das Alzheimerrisiko senken. Auch Menschen, die mediterrane Nahrung bevorzugen, werden seltener dement. Milchprodukte mit hohem Fettanteil, viel Fleisch und Butter erhöhen hingegen das Demenzrisiko.
\end{abstract}

- Wissenschaftler der Columbia University New York untersuchten pro-

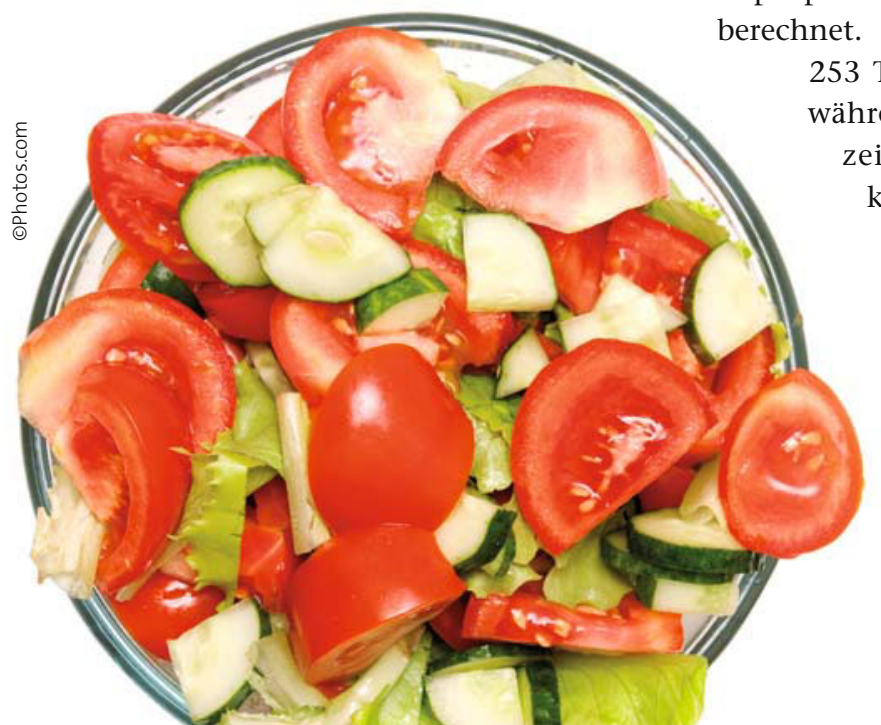

spektiv 2148Personen ab 65 Jahren aus Nord-Manhattan in Abständen von je 1,5 Jahren über insgesamt 3,9 Jahre im Mittel. Zu Studienbeginn zeigten sie keine Anzeichen einer Demenz. Per Fragebogen wurden die Ernährungsgewohnheiten der Probanden erfasst. Die kognitiven Leistungen wurden mit standardisierten neurologischen und neuropsychologischen Tests evaluiert. Die Assoziation der Ernährung mit der Alzheimerkrankheit wurde mit dem COX-proportionalen Hazardmodell 53 Teilnehmer erkrankten ährend der Beobachtungszeit an der Alzheimerkrankheit. Unter ihnen waren besonders viele, die sich fett- und fleischreich ernährt hatten. Teilnehmer, die mediterrane Kost zu sich nahmen, litten deutlich seltener an Demenz. Das geringste Alzheimerrisiko (HR
0,62) hatten Personen, deren Speiseplan viel Salat, Nüsse, Fisch, Tomaten, Geflügel, Wurzelgemüse und Blattgrün und dafür nur wenig fette Milchprodukte, rotes Fleisch, Innereien und Butter vorsah.

\section{Kommentar}

Wie die Studie zeigt, kann das Alzheimerrisiko durch eine gesunde Diät gesenkt werden. Das in der Studie ermittelte wirksamste Diätmuster könnte über drei Mechanismen protektiv wirksam werden: Vitamine senken den Homocysteinspiegel und/oder haben antioxidative Effekte. Und die fettarme Ernährung schützt vor Gefäßverkalkungen und -entzündungen, die ebenfalls zur Demenz beitragen.

K. MALBERG

\footnotetext{
- Y. Gu et al

(Columbia Medical Center, Cognitive Neuro-science Div, The Taub Institute, $630 \mathrm{~W}$ 168th Street, New York, 10032, USA; e-mail: yg@columbia.edu) Food combination and Alzheimer disease risk, a protective diet. Arch Neurol 2010; Published online: April 12, 2010; doi: 10.1001/archneurol.2010.84
} 